\title{
Wireless transmission and analysis of physical variations in the atmosphere using ZIGBEE
}

\author{
Jayalakshmi M Nair ${ }^{1}$, Sureshchandra J Gupta ${ }^{2,}$ Mily Jashank ${ }^{3}$ \\ ${ }^{I}$ (Electronics Department, Thakur Polytechnic, India) \\ ${ }^{2}$ (University Department of Physics, Mumbai University, India) \\ ${ }^{3}($ Information Technology Department, Thakur Polytechnic, India)
}

\begin{abstract}
During periods of background seismicity large variations in temperature occur due to crustal deformation. Earthquakes are preceded due to ground lengthening, tilting or shortening. A large amount of heat energy at the earth's core tries to find a path to come out from one way or the other. In this paper, we present an instrumentation system to study the variations in temperature that occur prior to an earthquake. The changes in the temperature and relative Humidity are sensed by using Smartec sensors SMT16030 and SMTHS07.The Smartec temperature sensor SMT16030 is a sophisticated active full silicon temperature sensor with a digital output. The capacitive humidity sensor SMTHSOTof Smartec is based on silicon technology on glass wafer. The sensors are powered by using solar panel. The output of the sensor is directly connected to a zigbee transceiver module. The data is further transmitted wirelessly over a distance of 120 metres by using Zigbee transceivers. ZigBee is an open global standard built on the IEEE 802.15.4 MAC/PHY. The received data is recorded and analyzed using PICI8F4520 microcontroller and further transmitted on to the Internet through the GPRS network.
\end{abstract}

Keywords: GPRS, PIC microcontroller 18F4520,Smartec temperature sensor SMT16030,SMARTEC Capacitive humidity sensor(SMTHSO7), Zigbee.

\section{INTRODUCTION}

Earthquake is one of the most prominent natural calamities on earth. In spite of the large amount of research done in this area worldwide, the methodology and instrumentation for accurate prediction of earthquakes (even a few hours before their occurrence) has remained elusive and not much success has been achieved. An Earthquake is a sudden and temporary movement of the Earth, ranging from a faint tremor to a wild motion, due to the sudden release of accumulated strain. The strain is released in the form of waves called Seismic waves. These waves cause vibrations while travelling through the Earth. These elastic waves or seismic waves are produced when some form of stored energy such as elastic strain, chemical energy, heat energy or gravitational energy is released suddenly[1]. The types of waves which exist before and during the earthquake are similar to those produced during a nuclear blast or an explosion inside the earth. Earthquakes are usually preceded with physical variations in atmosphere. Large variations in pressure, temperature and humidity occur much before and during the earthquake. A large amount of heat energy at the earth's core tries to find a path to come out from one way or the other [2]. The portion of earth's crust, which gets affected the most due to this, is the earthquake prone area.

In this paper, we present an instrumentation system to detect the variations in temperature and relative humidity that occur prior to an earthquake. The changes in the temperature are sensed [3] by using a Smartec temperature sensor SMT16030. The Smartec temperature sensor is a sophisticated active full silicon temperature sensor with a digital output. The one wire output (duty-cycle modulated) temperature sensor does not need A/D conversion. The temperature range is $-45^{\circ} \mathrm{C}$ to $150{ }^{\circ} \mathrm{C}$. The high resolution $\left(<0.005{ }^{\circ} \mathrm{C}\right)$ makes the sensor useful for high precision applications. The sensor is powered by using solar panel. The output of the temperature sensor is directly connected to a Zigbee router. Measuring of humidity in the atmosphere is difficult. Generally the humidity in air is measured as the fraction of the maximum amount of water that air can absorbed at a certain temperature. At atmospheric conditions and a given temperature this fraction can vary between 0 (absolute dry) and $100 \%$ (the point where condensation will begin to form). This relative humidity is only valid at a certain temperature and atmospheric pressure. Therefore it is important that a humidity sensor should not be affected by either temperature or pressure. The capacitive humidity sensor of Smartec is based on silicon technology on glass wafer. It is a two terminal capacitor, which increases in value as water molecules are absorbed into its active polymer dielectric. The capacitor plates consist of a base plate and a water permeable platinum top plate. The sensor converts the relative humidity into a capacitance. Due to the construction, the response to humidity is very fast $(<15 \mathrm{sec})$ and the hysteresis very low $(<2 \% \mathrm{RH})$. The humidity sensor is connected to an astable multivibrator to generate the required digital signal which acts as an input to the zigbee router. 
The sensed data ie temperature and relative humidity is further transmitted wirelessly over a distance of 120 metres by using Zigbee transceivers. The zigbee with whip antenna transmits the sensed data. ZigBee is an open global standard built on the IEEE 802.15.4 MAC/PHY. It requires a supply voltage in the range of 2.8V to $3.3 \mathrm{~V}$.The main advantage of using a wireless sensor is that it can be used with ease in the rugged terrain where it is impossible to use wired system. At the receiving end the zigbee coordinator receives the data. The received data is recorded and analyzed using PIC18F4520 microcontroller and further transmitted on to the Internet through the GPRS network.The PIC18F4520 family introduces design enhancements that make these microcontrollers a logical choice for many high-performances, power sensitive applications. The Enhanced Flash cells for both program memory and data EEPROM are rated to last for many thousands of erase/write cycles - up to 100,000 for program memory and 1,000,000 for EEPROM. Data retention without refresh is conservatively estimated to be greater than 40 years. These devices can write to their own program memory spaces under internal software control. By using a bootloader routine located in the protected Boot Block at the top of program memory, it becomes possible to create an application that can update itself in the field.

\section{Design And Development}

Several studies have shown that there is an increase in temperature of 4-8 degree Celsius few days prior to big earthquakes. From the study it has been statistically proved that the moisture holding capacity of air depends on the air's temperature. It increases with increase in temperature. As the moisture holding capacity increases the relative humidity decreases, provided no moisture is added to the air. This conclusion will help in better understanding of the relationship between temperature and moisture holding capacity of the air and relative humidity. In this paper we have designed and developed an experimental system capable of sensing the changes in temperature and relative humidity of a remote location and logging it on the internet using the GPRS intelligent Modem after a detailed analysis of the sensed value. The experimental study is split up into several steps.

\subsection{Basic Block Diagram of the Wireless Monitoring System}

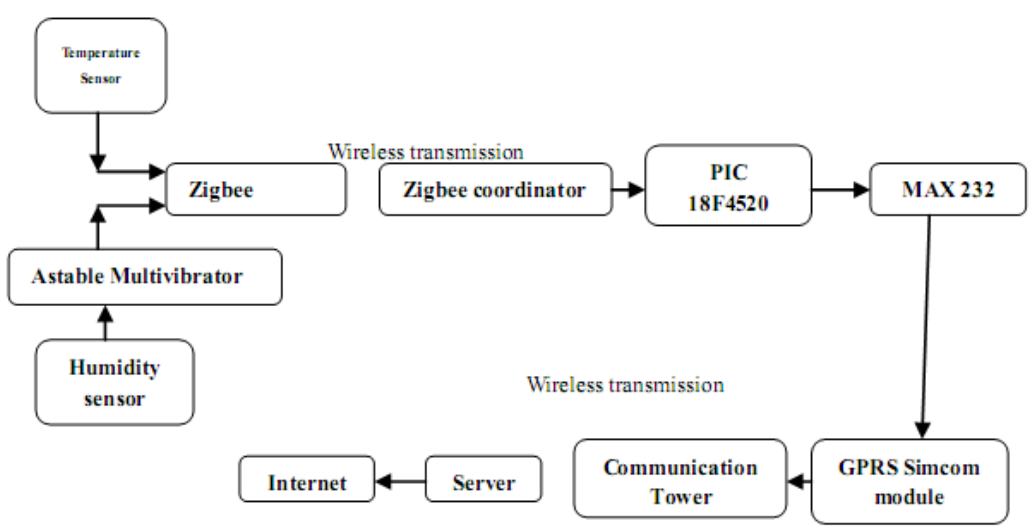

Figure 1 Block diagram of the wireless Monitoring System

Figure 1 shows the basic block diagram of the Wireless Monitoring System. It consists of temperature and humidity sensor, zigbee transceiver module, solar powered DC supply, PIC 18F4520, MAX RS 232, GPRS Module.

\subsubsection{The Smartec temperature sensor}

It is a sophisticated full silicon temperature sensor with a digital output. The temperature range is $-45^{\circ} \mathrm{C}$ to $150{ }^{\circ} \mathrm{C}$. The high resolution $\left(<0.005^{\circ} \mathrm{C}\right)$ makes the sensor useful for high precision applications. The integrated modulator ensures the sensor unit can communicate effectively with lowcost microcontrollers without the need of (onboard) A/D converters or an Xtal controlled oscillator. The SMT160-30 is a three terminal integrated temperature sensor. Two terminals are used for the power supply of 5 Volts and the third terminal carries the output signal. This sensor is especially suited for applications where very accurate measurements are needed. The CMOS output of the sensor can handle cable length up to 20 meters. The output is a square wave with a well-defined temperaturedependent duty cycle. 


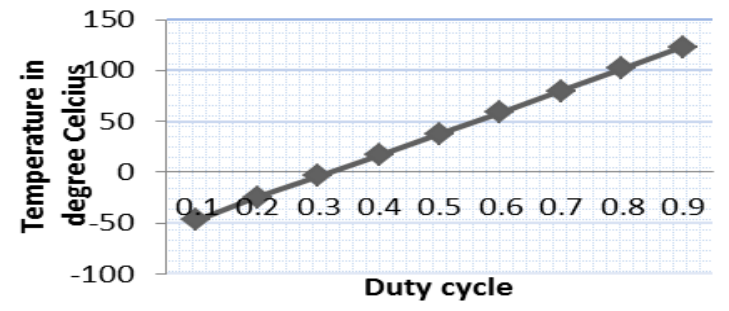

Fig 2: Graph of temperature Versus DutyCycle

The duty cycle of the output signal is linearly related to the temperature according to the equation:

D. $C=0.320+0.00470 x t$

D.C. $=$ duty cycle

$t=$ Temperature in ${ }^{\circ} \mathrm{C}$

In the output frequency of the sensor there is no temperature information, only the duty cycle contains temperature information in accordance to the formula given above. The sensor output is directly connected to pin no 20 i.e.AD0 / DIO0 of the Zigbee router.

\subsubsection{The Smartec humidity sensor (SMTHS07)}

It is a two terminal capacitor, which increases in value as water molecules are absorbed into its active polymer dielectric. The capacitor plates consist of a base plate and a water permeable platinum top plate. Besides the active dielectric, polymers give protection to the base plate, prevent polarization and pin holing and generally ruggedize the sensor so that it can be used in a wide range of hostile environments. The Smartec capacitive humidity sensor measures from 0 to $100 \% \mathrm{RH}$ and operating temperature is -40degree $\mathrm{C}$ to 120 degree $\mathrm{C}$. The change in the relative humidity is measured as capacitance. The humidity sensor is connected to a typical astable multivibrator TLC 555 (CMOS type). The SMTHS07 is used as variable capacitor. The output voltage varies between $0.5 \mathrm{Vcc}$ and Gnd. The output of the multivibrator is directly connected to pin no 19 ie $\mathrm{AD} 1$ / DIO1 of the Zigbee router.

\subsubsection{ZIGBEE transceiver module:}

Zigbee is a high level communication protocol using small, low-power digital radios based on the IEEE 802.15.4 standard for wireless networks. A Zigbee can have up to several hundred (client) nodes per network,16 channels in the global $2.4 \mathrm{GHz}$ band, Full Mesh Networking Support,128bit AES security Network and link keys can restrict access. It is easily deployable, Reliable - self forming \&self-healing and has very long battery life at a very low cost. Data transmission rates vary from 20 kilobits/second in the $868 \mathrm{MHz}$ frequency band to 250 kilobits/second in the $2.4 \mathrm{GHz}$ frequency band. It needs to be configured through XCTU software. In the present instrumentation system, XBee -Pro RF modules had been used to cover a good range for wide application areas. XBee- Pro is having the indoor range of $90 \mathrm{~m}$ and outdoor line of site up to 1 mile. The ZigBee RF Modules interface to a host device through a logic-level asynchronous serial port.

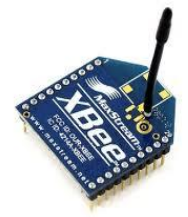

Fig 3: Zigbee with whip antenna

Serial Transmission: Data enters the ZIGBEE router through the pins 20(temperature) and 19 (relative humidity). The signal should idle high when no data is being transmitted. Each data byte consists of a start bit (low), 8 data bits (least significant bit first) and a stop bit (high). The ZigBee modules maintain small buffers to collect received serial data. The serial receive buffer collects incoming serial characters and holds them until they can be processed. The serial transmit buffer collects data that is received via the RF link that will be transmitted out the USART. 


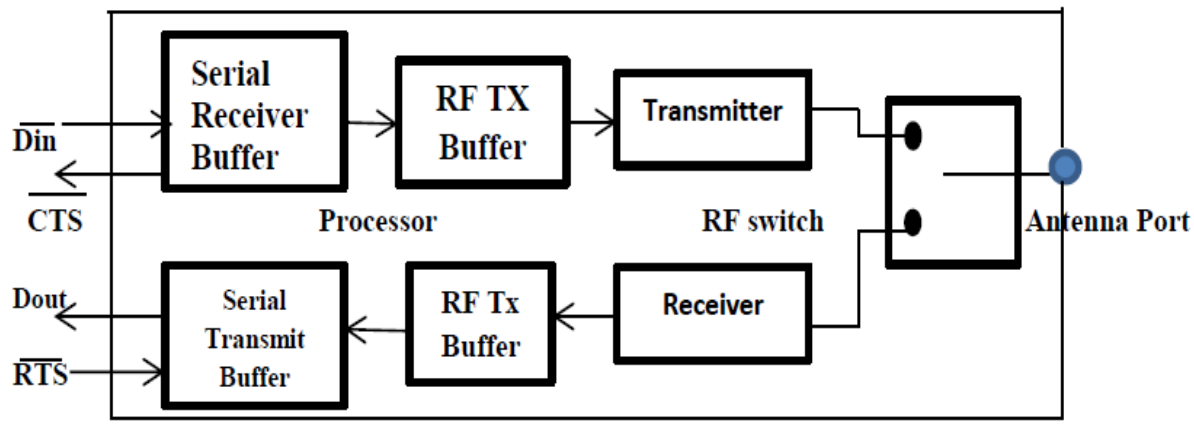

Fig 4: Internal Data Flow Diagram

When serial data enters the RF module the data is stored in the serial receive buffer until it can be processed. Under certain conditions, the module may not be able to process data in the serial receive buffer immediately. If large amounts of serial data are sent to the module, CTS flow control may be required to avoid overflowing the serial receive buffer. When RF data is received, the data is moved into the serial transmit buffer and sent out the USART. Prior to transmitting the data, the module ensures that a 16-bit network address and route to the destination node have been established [6].

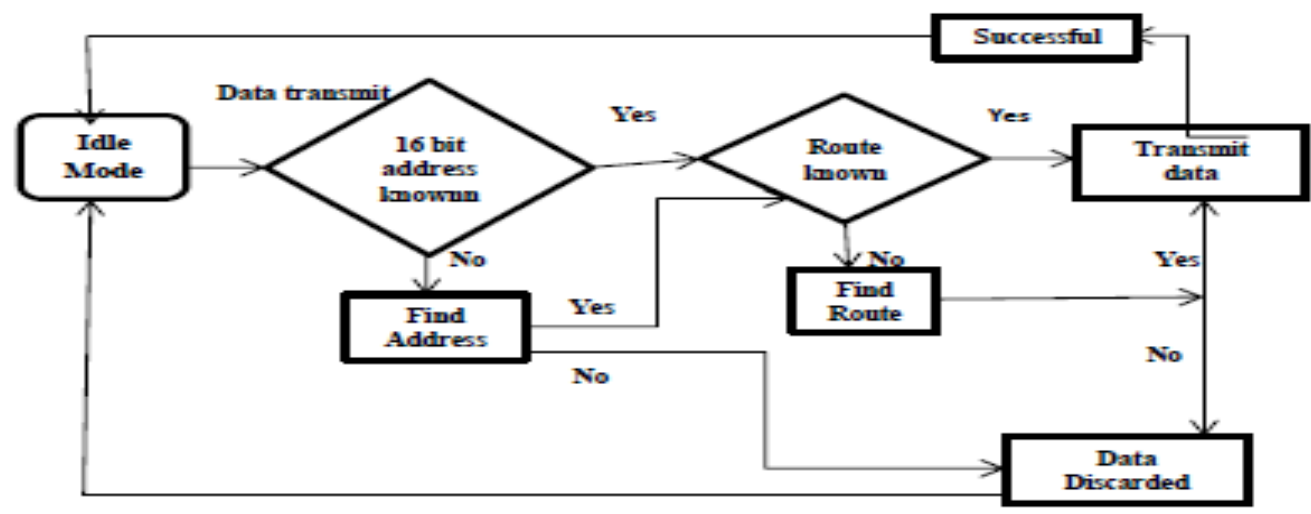

Fig 5-Data Transmission in Zigbee

The Zigbee coordinator at the receiving side receives the transmitted data. If a valid RF packet is received, the data is transferred to the serial transmit buffer. The Tx pin (pin no 2) of Zigbee coordinator is connected to the pin $26(\mathrm{Rx})$ of PIC18F4520 for further analysis of the detected temperature and relative humidity.

\subsubsection{PIC 18F4520}

The PIC18F4520 offers many new features, including extended instruction set, power management modes, higher speed ADC, Enhanced USART and Enhanced CCP mode. These modes provide a variety of options for selective power conservation in applications where resources may be limited. In addition to the Run and Sleep modes, the PIC18F4520 also offers the Idle mode of operation. The Idle mode allows the controller CPU to shut-down selectively while the peripherals continue to operate. The ECCP module is implemented as a standard CCP module with Enhanced PWM capabilities. These include the selection of 1, 2 or 4 PWM outputs, user selectable polarity, dead-band control and automatic shut-down and restart. By default, the ECCP module is configured in Single Output mode and functions identically to the standard CCP module in PWM mode. The USART module in the PIC18F4520 is implemented as EUSART (Enhanced USART). The Enhanced USART module implements additional features, including Automatic Baud Rate Detection and calibration, automatic wake-up on Sync Break reception and 12-bit Break character transmit. The Baud Rate Generator (BRG) in the PIC18F4520 is a dedicated 8-bit or 16-bit generator that supports both the Asynchronous and Synchronous modes of the EUSART. The Fail-Safe Clock Monitor allows the microcontroller to continue operation in the event of an external oscillator failure by automatically switching the device clock to the internal oscillator block. By default, the Fail-Safe Clock Monitor is disabled. The nominal WDT period in the PIC18F4520 is 4 ms .If the WDT option is enabled, the user needs to select the appropriate WDT time-out period for the PIC18F4520.The PIC18F4520 provides Enhanced ICD features which include three Break points and Break on GPR address and data match[5]. 
A) Smartec temperature sensor: The PIC 18F4520 microcontroller determines directly determines the duty cycle value of the temperature sensor by sampling the sensor output. The sensor generates a square wave signal. The amplitude is the same as the input voltage $(5 \mathrm{~V})$, the frequency varies between $1 \mathrm{kHz}$ and $4 \mathrm{kHz}$.

The duty cycle is then converted to degree Celsius by using the formula:

Temperature $=\frac{\text { Duty cycle }-0.32}{0.0047}$

Where,

D. $C=0.320+0.00470 x t$

D.C. $=$ duty cycle

$\mathrm{t}=$ Temperature in ${ }^{\circ} \mathrm{C}$.

B) Smartec humidity sensor: The humidty sensor detects the changes in the relative humidity in the form of capacitance. The relative humidity can be calculated as

$\mathrm{Xrh}=(\mathrm{Cc}-\mathrm{Cs}) / \mathrm{S}+55$

Where,

$\mathrm{Cc}=$ measured capacitance $(\mathrm{pF})$

$\mathrm{Cs}=$ capacitance value at $55 \% \mathrm{RH}(\mathrm{pF})$

$\mathrm{Xrh}=$ measured Relative Humidity $(\%)$

$\mathrm{S}=$ sensitivity

The Humidity sensor is then connected to an Astable Multivibrator to convert the sensed capacitance to a corresponding digital pulse. The PIC 18F4520 microcontroller determines the frequency by using the formula: $\mathrm{f}=1.44 /(\mathrm{C} 1(\mathrm{R} 1+2 \mathrm{R} 2))$

where $\mathrm{C} 1$ is the sensed capacitance of the humidty sensor.

\subsubsection{GPRS Transmission}

The temperature and the relative humidity readings is read using the HyperTerminal. Hence the variations in the temperature and the relative humidity detected by the sensor can now be seen on the Computer with the help of connection-oriented circuitry (serial cable). After verifying that the readings are in accordance to the variations of the temperature and relative humidity, our next task was to transmit the data between the PIC18F4520 and the PC, by using wireless techniques instead of the Serial Cable. The data is then transmitted by using GPRS (General Packet Radio Service) network on to the Internet through the GPRS Intelligent Modem (GIM) also called as GPRS module This particular GPRS MODEM is called Intelligent MODEM since it contains a PIC (Programmable Interface Controller) - microcontroller. For GPRS transmission, the data to be transmitted should be in the ASCII HEX form. Hence the data has to be converted to its ASCII HEX equivalent value before transmitting. This is accomplished by modifying the software program of the PIC18F4520. To transmit via GPRS, the user should first get the access to the GPRS Gateway. To get this access one needs an Access Point Name (APN). The user gets the APN only when it uses a DATA SIM CARD or GPRS DATA SIM CARD[7]. The SIM (Subscriber Identity Module) CARD provided by the Service Provider will ultimately provide the Access Point Name to get to the GPRS Gateway. This SIM CARD is to be housed in the GPRS module. In our set-up, we have used Vodafone's DATA SIM CARD.

In order to read the transmitted data which is in the ASCII HEX form into readable form, a program written in PERL language is stored in the User's Server. As soon as the data reaches the port of the User's Server, the program which is written in PERL language is executed i.e. a .PL file program is executed. After the execution of the .PL file program, the final result is stored in the memory of the User's Server in the form of database required by the user[8]. Each time the data is transmitted; this will be stored in the memory of the User's Server in the form of a file. Now whenever the User accesses the particular Website and calls this file the data will be displayed on the monitor of the PC. Along with the data the User can also read the Time, Day and Date on which the data was registered by the User's Server. Each time the data is received by the User's Server, the file in the User's Server is updated without erasing the previous records. The User can thus monitor the change in temperature of a particular area (site) by just visiting the particular Website[8].

\section{Results:}

A) The Smartec temperature sensor is a sophisticated full silicon temperature sensor with a digital output. The SMT160-30 is with accurate electronics converts the sensor signal into a duty cycle. The output is a square wave with a well-defined temperature-dependent duty cycle. The duty cycle of the output signal is linearly related to the temperature. The PIC18F4520 microcontroller determines the duty cycle value by sampling the sensor output.

B) The Smartec humidity sensor converts the humidity into a capacitance. The Smartec capacitive humidity sensor measures from 0 to $100 \% \mathrm{RH}$. An astable multivibrator converts the capacitance to a digital signal. 
The Zigbee router with whip antenna transmits this data. The Zigbee coordinator at the receiving side receives the transmitted data and gives it to PIC 18F4520.The microcontroller analyses the data and sends the data to the PC via MAX 232.The data is then converted to ASCII HEX equivalent value for wireless transmission through GPRS.

\section{Conclusions}

A) A wireless electronic instrumentation system to measure the variations in the temperature and relative humidity is designed and developed. A digital sensor SMARTEC SMT 16030 and humidity sensor SMTS07 is interfaced to wireless ZIGBEE Transceivers .The sensor is powered using solar panel. The temperature and the relative humidity fluctuations at the remote location are analyzed and monitored on the PC (HyperTerminal) in the Control Room (laboratory) which is $120 \mathrm{mts}$ away from the site area.

B) These temperature and relative humidity fluctuations are also monitored with wireless systems using GPRS network on to the internet using GPRS Intelligent Modem.

\section{Acknowledgments}

Our sincere thanks to the experts Dr S.K Dubey, Dr D.C Kala and Dr H.S Kalsi who have contributed towards development of this paper.

\section{References}

[1] Peter M Shearer, Introduction to Seismology, (Cambridge University Press, Cambridge, 1999) p.p.(20-31).

[2] Alok K Gupta, Somnath Dasgupta, Physics and Chemistry of Earth's interior crust mantle and core, (Springer India, 2009) p.p ( 323).

[3] Kalsi H.S, Electronic Instrumentation, (McGraw Hill Publication, 2004) p.p.(425-446).

[4] Jan Axelson, The Microcontroller idea book: Circuits program and Applications, (Penram international, India, 1994) p.p (153166).

[5] Mazidi M.A,Mazidi J, Pic Microcontroller and Embedded Systems, (Pearson Education, India p.p (187-194).

[5] Geoff Sanders,Lionel Therons, GPRS Networks, (John Wiley and Sons, 2009).

[6] Nair J.M,Gupta S.J,Jashank M, GPRS Transmission of Onsite Temperature Variations, Proc. International conference \& Workshop on emerging trends in technology, India, 2013, 289-293. 